\title{
CAR T cells drive out $B$ cells in SLE
}

The treatment
resulted in
complete and
sustained
CD $19^{+}$B cell
depletion

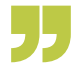

$B$ cell depletion is an effective approach for the treatment of several diseases, including rheumatoid arthritis (RA); however, despite showing initial promise in the treatment of systemic lupus erythematosus (SLE), the B cell depleting antiCD20 antibody rituximab did not reach the clinical endpoints in two large phase III clinical trials. Almost a decade later, a promising new approach to $B$ cell depletion in SLE is emerging: CD19-targeted chimeric antigen receptor (CAR) $\mathrm{T}$ cell therapy.

"The methodology involves inserting an artificial gene into the chromosomes of the patients' own T cells," explains Marko Radic, corresponding author of the new study published in Science Translational Medicine. "The new gene encodes a CAR that targets CD19 on the majority of $B$ cells and kills them upon binding."

Unlike a standard CAR T cell approach, the researchers used purified $\mathrm{CD} 8^{+} \mathrm{T}$ cells to avoid any potential disease-promoting effects that might come from using $\mathrm{CD} 4^{+}$ $\mathrm{T}$ cells. As a second generation $\mathrm{CAR}$, the $\mathrm{CD} 3 \zeta \mathrm{C}$ terminus had been modified to dampen its activity and prevent $\mathrm{T}$ cell exhaustion (increasing the persistence of this therapy).

The CAR T cell approach avoids the need for repeated administration, as is necessary for rituximab to retain therapeutic doses, and could potentially lead to longlasting remission. In previous trials of rituximab, the degree of $B$ cell depletion was variable, but those who had the most rapid, largest or longest-lasting B cell depletion had the most improvement in disease presentation. Hence, incomplete or only transient depletion of tissue-resident $B$ cells might have contributed to the lack of efficacy of rituximab in SLE trials.

"Compared with ritixumab, the new approach has two main advantages," remarks Ignacio Sanz, an expert in anti-B cell therapies in SLE who was not associated with the study. "Firstly, anti-CD19 targeting eliminates a larger fraction of B cells, including pre-B cells, as well as a large fraction of antibody-producing plasmablasts and plasma cells. Secondly, antiCD19 CAR T cells, as reported in lymphoid malignancies, can induce B cell depletion of greater depth and duration."

Radic and colleagues tested the efficacy of this new approach in two mouse models of SLE: NZB/W and MRL-lpr mice. The treatment resulted in complete and sustained $\mathrm{CD} 19^{+} \mathrm{B}$ cell depletion that was accompanied by a reduction in autoantibody production, "The treated mice showed considerable improvements in several measures of disease, including improvements in kidney function, spleen size, lymphocyte subset ratios and skin inflammation," says Radic.

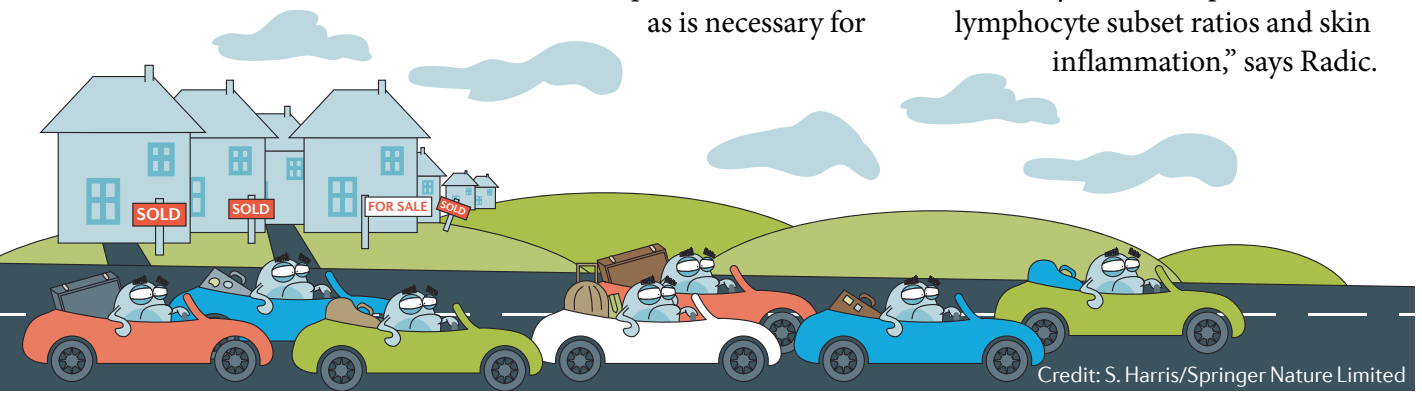

"Most importantly, treated mice attained a nearly normal life span."

Although the treatment effectively diminished the expression of CD19 in the kidneys, spleen and bone marrow, the expression of immunoglobulin light chains persisted in the spleen and bone marrow, indicating the continued presence of some B cells at these sites that might explain the preserved levels of IgM and IgG antibodies in the serum.

As further evidence of the persistence of this therapy, adoptively transferred B cells were effectively depleted in mice that had already received CAR T cell therapy months previously. In a separate experiment, adoptive transfer of $\mathrm{T}$ cells from treated mice to untreated recipient mice induced B cell depletion and reduced disease activity, indicating that the effects of this therapy are cell-mediated.

"Progress with CAR T cells in cancer over the past few years and the FDA approval of this therapy in 2018 indicate that the use of CAR T cell therapy is maturing and reaching a level of safety and acceptability in the clinic that might make it attractive to rheumatologists," concludes Radic.

"This work provides a strong rationale for the use of CAR $\mathrm{T}$ cells for the treatment of human autoimmune diseases and, in particular, for SLE," says Sanz. "The natural next step in the field is the design of human clinical trials to assess the safety and efficacy of this approach in SLE and other autoimmune diseases including ANCAassociated vasculitis, in which repeated rituximab treatments might be necessary to sustain disease remission."

Jessica McHugh

ORIGINAL ARTICLE Kansal, R. et al. Sustained B cell depletion by CD19-targeted CAR T cells is a highly effective treatment for murine lupus. Sci. Transl. Med. 11, eaav1648 (2019) 